\title{
Satisfacción laboral y productividad en docentes de la Facultad de Enfermería de la UNCP. Huancayo, 2016
}

\section{Job satisfaction and productivity in teachers of the Faculty of Nursing of the UNCP. Huancayo, 2016}

\author{
'Pérez Gutarra, Guillermina Delia; Scarsi Maratuech, Rossana Mirt; Merino Lozano, Alicia Lourdes, \\ ${ }^{2}$ Habich Scarsi, Bruno \\ Facultad de Enfermería, Universidad Nacional del Centro del Perú \\ Email: gperezg@uncp.edu.pe
}

\section{Resumen}

Un tema de gran interés para los investigadores en gestión es la relación entre la satisfacción y productividad. La pregunta habitual es si los trabajadores satisfechos son más productivos que los insatisfechos, se entiende que la productividad es un indicador de la eficiencia y competitividad de la institución. El objetivo general de la presente investigación es: Determinar la relación de la satisfacción laboral y la productividad de docentes de la Facultad de Enfermería de la UNCP. La muestra fue 26 docentes de la Facultad de Enfermería a quienes se les aplicó el cuestionario de satisfacción laboral y el cuestionario de productividad. Siendo las conclusiones: Los docentes de la Facultad de Enfermería, en su mayoría (38\%), tienen un nivel medio de satisfacción laboral, y el $30 \%$ tienen un nivel bajo de satisfacción frente al $30 \%$ que tiene un nivel alto de satisfacción laboral. La productividad en investigación de los docentes de la Facultad de Enfermería es bajo; si bien se realiza investigaciones anualmente, la debilidad está en la publicación de los mismos en revistas indexadas o de prestigio, siendo escasa o nula la publicación en muchos casos. Se ha comprobado que no existe relación entre la satisfacción laboral de los docentes y la baja productividad que tienen, independientemente del nivel de satisfacción la mayoría tienen baja productividad.

Palabras Claves: satisfacción, productividad, producción científica

\begin{abstract}
One issue of great interest to management researchers is the relationship between satisfaction and productivity. The usual question is whether the satisfied workers are more productive than the dissatisfied, it is understood that productivity is an indicator of the efficiency and competitiveness of the institution. The general objective of the present investigation is: determining the relation of the labor satisfaction and the productivity of teachers of the Nursing Department of the UNCP. The sample consited of 26 teachers from the nursing department who answered the job satisfaction questionnaire and the productivity questionnaire. The conclusions were: the lecturers of the Nursing Department (38\%) have an average level of job satisfaction, and 30 $\%$ have a low level of satisfaction compared to $30 \%$ who have a high level of job satisfaction. The research productivity of nursing department is low, although annual researches are carried out, the weakness lays in the publication of the same in indexed or prestigious journals, with little or nil publication in many cases. It has been verified that there is no relation between lecturers' job satisfaction and the low productivity they have; regardless of the satisfaction level, the majority have low productivity.
\end{abstract}

Keywords: satisfaction, productivity, scientific production 


\section{Introducción}

Un tema de gran interés para los investigadores en gestión es la relación entre la satisfacción y productividad. La pregunta habitual es si los trabajadores satisfechos son más productivos que los insatisfechos (Robbins, 1998). Se entiende la productividad como la medida de qué tan bien funciona el sistema de operaciones o procedimientos de la organización. Es un indicador de la eficiencia y competitividad de la organización o de parte de ella (Stoner, 1994).

En relación a la productividad del docente, se puede mencionar que está referida a la producción intelectual investigativa, lo que se convierte en un factor de éxito en la formación profesional de calidad. Ambos elementos son necesarios para el desarrollo universitario en el marco de los desafíos y exigencias actuales.

El objetivo general planteado es: Determinar la relación de la satisfacción laboral y la productividad de docentes de la Facultad de Enfermería de la UNCP. Como objetivos específicos se tiene: Identificar el nivel de satisfacción laboral en docentes de la Facultad de Enfermería de la UNCP; e Identificar el nivel de productividad en docentes de la Facultad de Enfermería de la UNCP.

En la presente investigación, se toma la teoría bifactorial propuesta por Frederick Herzberg, en la medida que se articula a los objetivos propuestos. La Producción en investigación es la forma mediante la cual una universidad o institución se hace presente a la hora de hacer ciencia; sin embargo, se sabe que una investigación científica no concluye hasta que no se han publicado sus resultados, por tanto, para hacer ciencia también hay que escribir ciencia. Pinilla (1982), plantea una relación muy importante entre la satisfacción laboral y la productividad; él dice: «por satisfacción se entiende la actitud general que adoptamos frente a nuestro trabajo, cuando hemos podido resolver nuestra necesidades fundamentales y tenemos conciencia de ello, en tal sentido, los trabajadores necesitan que se les respete y se les trate dignamente. Necesitan ganar lo suficiente para vivir decorosamente, para alimentarse, vestirse y tener recreación, pero no sólo el individuo, sino también su familia.

\section{Materiales y métodos}

El presente estudio es de tipo cuantitativo, correlacional de corte transversal. La población estuvo conformada por 33 docentes y la muestra fue de 26 docentes que viene a ser el $78 \%$ de la población.

El diseño de investigación, por ser un estudio de tipo correlacional transversal; responde al siguiente esquema:

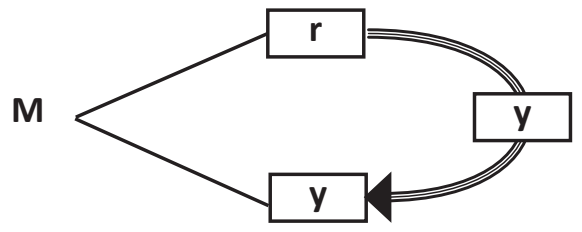

\section{Dónde:}

$\mathbf{x}=$ Conjunto de datos con respecto a satisfacción laboral

$\mathbf{y}=$ Conjunto de datos con respecto a productividad

$\mathbf{r}=$ Relación existente entre ambas variables

\section{Técnica e instrumentos de recolección de datos}

Para medir la satisfacción laboral de docentes de la Facultad de Enfermería de la UNCP, se utilizó la técnica encuesta con el instrumento cuestionario del autor David Marcial Álvarez Flores - Lima 2003. La validez del instrumento que realizó el autor fue a través de juicio de expertos, siendo seleccionados 10 jueces expertos en el tema, la concordancia fue alta considerándose valido el instrumento. La confiabilidad la estimó mediante el análisis de consistencia interna utilizando el coeficiente Alfa de Cronbach obteniéndose 0,93.

Para medir la productividad de los docentes se elaboró el instrumento cuestionario tomando como referencia el instrumento de Pasache Herrera y Torres Vásquez (2011); así como, el de Escobar, García y Larrán (2013). El instrumento fue validado por juicio de expertos ( 8 jueces) existiendo concordancia entre los jueces, por lo tanto, se considera válido el instrumento, luego se realizó la prueba piloto encuestando a 20 docentes de otras facultades, sometiendo al coeficiente Alfa Cronbach, siendo el resultado 0,7284 , lo que significa que el instrumento es confiable.

\section{Resultados}

Los resultados obtenidos en el presente estudio permiten generar conocimientos para la implementación de estrategias y mejorar aspectos encontrados como debilidades. Los resultados están organizados según las variables de estudio.

\section{Dimensión: Satisfacción laboral}

\section{Gráfico 1}

Nivel de satisfacción laboral del personal docente de la Facultad de Enfermería de la UNCP - 2016. NIVEL DE SATISFACCIÓN

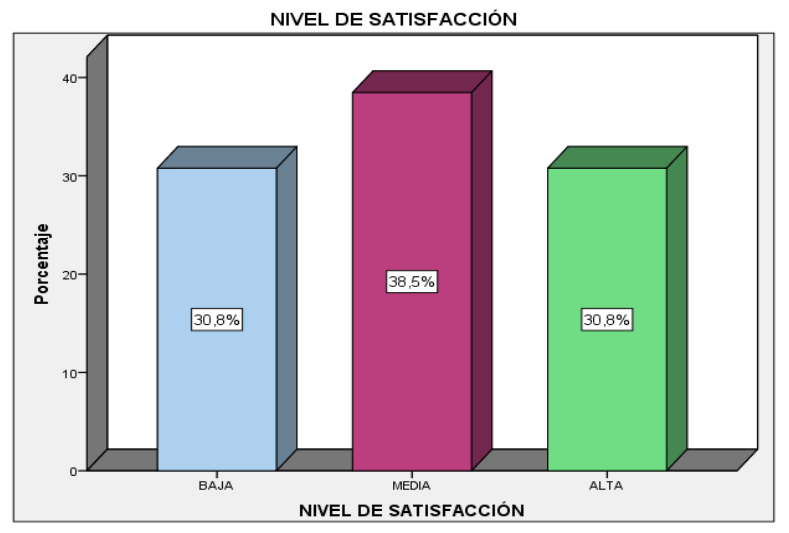

Fuente: Cuestionario satisfacción laboral docente de la FENUNCP 2016.

La satisfacción laboral del personal docente de la Facul- 
tad de Enfermería, muestra que el $30.8 \%$ la consideran baja y una ponderación igual (30.8\%), alta. En cuanto a la satisfacción media la percepción es de un $38.5 \%$ de docentes. Los resultados encontrados nos permiten inferir que no podemos tener una concepción de educación superior sino tenemos en cuenta la satisfacción laboral del docente universitario, para que pueda desempeñarse adecuadamente en el marco de los desafíos y exigencias actuales.

\section{Gráfico 2}

Nivel de satisfacción intrínseca personal docentes de la Facultad de Enfermería de la UNCP - 2016.

NIVEL DE SATISFACCIÓN INTRINSECA

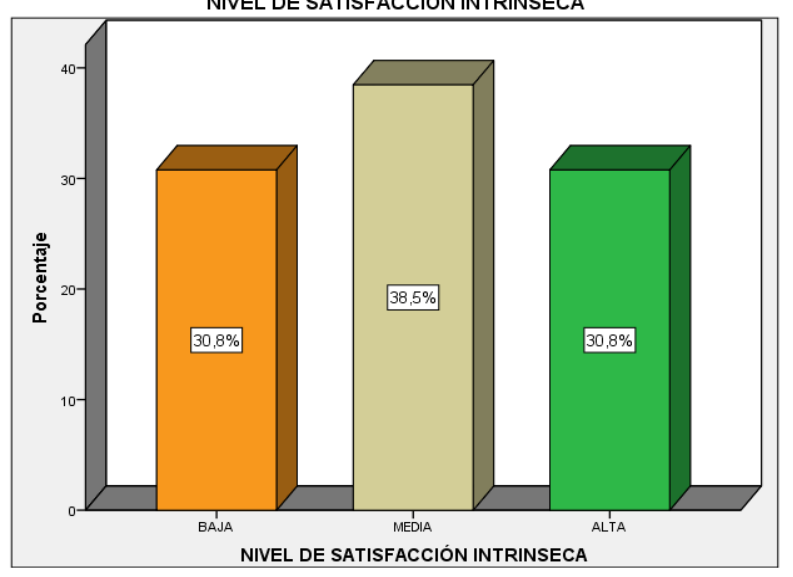

Fuente: Cuestionario satisfacción laboral docente de la FENUNCP 2016.

La dimensión de la satisfacción intrínseca incluye indicadores relacionados a: la motivación de preparar clases con los materiales que al docente le parecen más convenientes; si la institución propicia mecanismos de capacitación y desarrollo y; libertad para que el docente pueda elegir la metodología apropiada de su asignatura.

\section{Dimensión: Independencia}

\section{Gráfico 3}

Nivel de independencia del personal docente de la Facultad de Enfermería de la UNCP 2016.

NIVEL DE INDEPENDENCIA

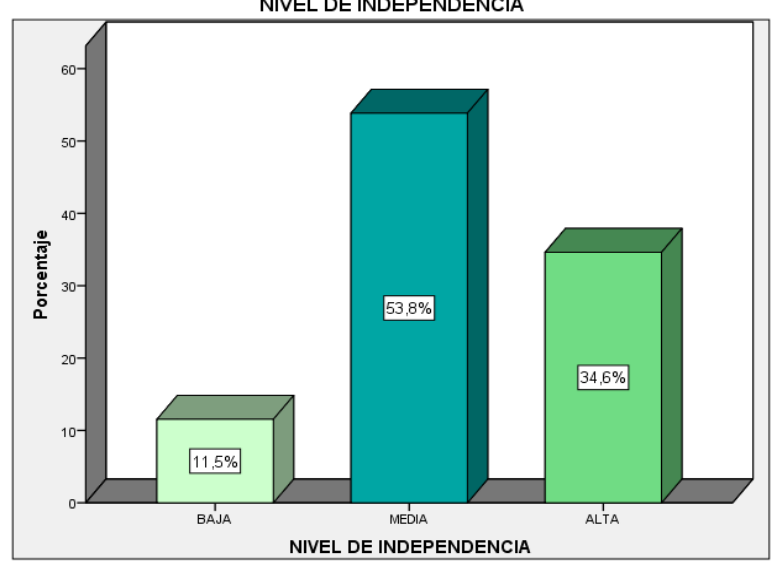

Fuente: Cuestionario satisfacción laboral docente de la FENUNCP 2016.
Se puede observar que el $34.6 \%$ de docentes consideran su satisfacción alta y un $53.8 \%$ media en relación a la dimensión independencia.

\section{Dimensión: Libertad de cátedra}

\section{Gráfico 4}

Nivel de la dimensión libertad del personal docente de la Facultad de Enfermería de la UNCP 2016. NIVEL LIBERTAD DE CÁTEDRA

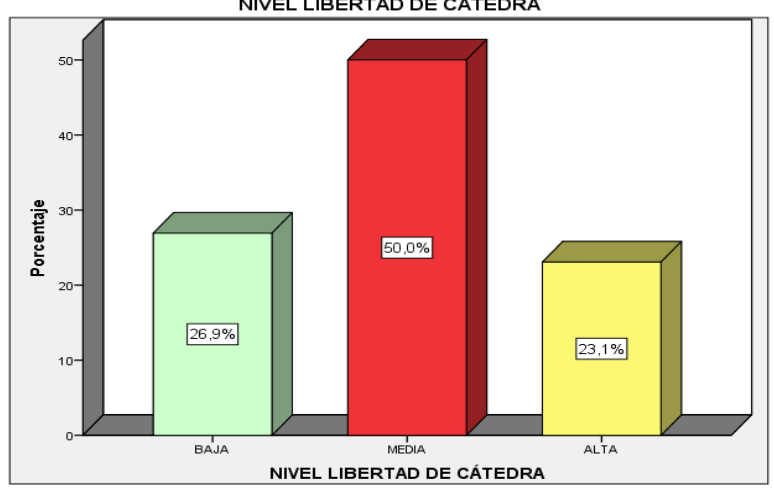

Fuente: Cuestionario satisfacción laboral docente de la FENUNCP 2016.

Los resultados nos muestran que un $26.9 \%$ de docentes refieren una satisfacción baja y $50 \%$ media en relación a su derecho de libertad de cátedra, lo cual está en estrecha relación a la asignación de las asignaturas; desacuerdo en que se les encargue mayor trabajo académico por su capacitación actual; temor para asumir cursos de alta especialización $\mathrm{y}$; perdida de autorrealización dictando cursos que les desagradan.

\section{Dimensión: Reconocimiento y distinciones}

\section{Gráfico 5}

Reconocimiento y distinciones del personal docente de la Facultad de Enfermería de la UNCP 2016. NIVEL DE RECONOCIMIENTO Y DISTINCIONES

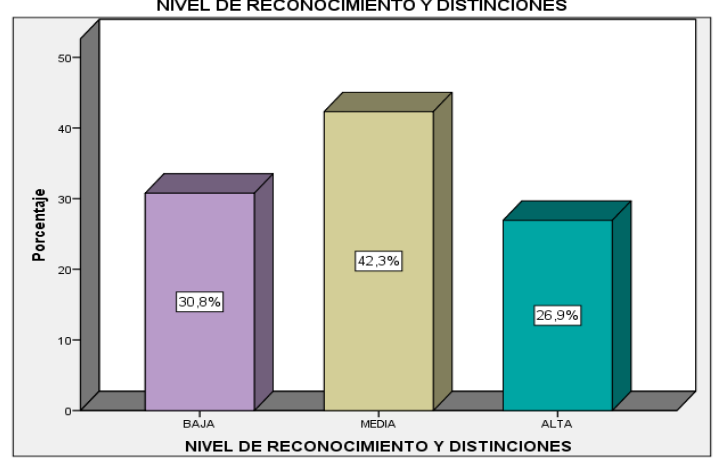

Fuente: Cuestionario satisfacción laboral docente de la FENUNCP 2016.

En relación a la dimensión reconocimiento y distinción del personal docente de la Facultad de Enfermería; más de la mitad de ellos consideran que es baja y media (30.8 $\%$ y $42.3 \%$ respectivamente), lo que nos hace inferir la insatisfacción de la mayoría de ellos en relación a que la facultad no premia sus esfuerzos los cuales pasan desa- 
percibidos y que los premios muchas veces se otorguen a allegados de autoridades.

\section{Factor: Satisfacción extrínseca}

\section{Gráfico 6}

Nivel de satisfacción extrínseca personal docentes de la Facultad de Enfermería de la UNCP 2016.

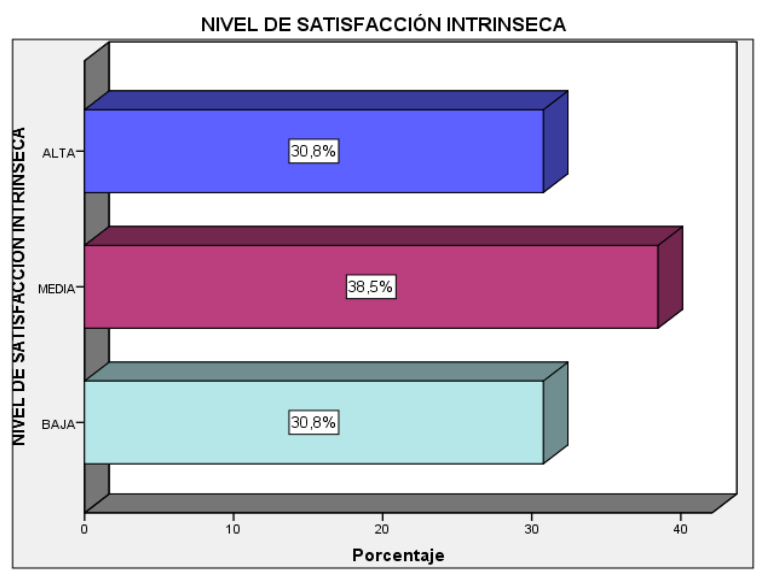

Fuente: Cuestionario satisfacción laboral docente de la FENUNCP 2016.

Los resultados globales de la satisfacción laboral extrínseca nos muestra una gran diversificación en las opiniones de los docentes de la Facultad de Enfermería. Con ponderaciones muy cercanas, opinan que su grado de satisfacción es baja, media y alta $(30,8 \% 38,5 \%$ y 30,8 $\%$ respectivamente). La insatisfacción extrínseca, es una fuente continua de insatisfacciones donde los docentes sienten mermadas sus posibilidades de expansión personal o sus deseos profesionales, lo que depende en gran medida, de las condiciones laborales y de la personalidad de cada uno y cuyos resultados influyen significativamente en la cultura y clima organizacional.

\section{Dimensión: Compensación económica}

\section{Gráfico 7}

Nivel de la dimensión compensación económica del personal docente de la Facultad de Enfermería de la UNCP 2016 NIVEL COMPENSACIÓN ECONÓMICA

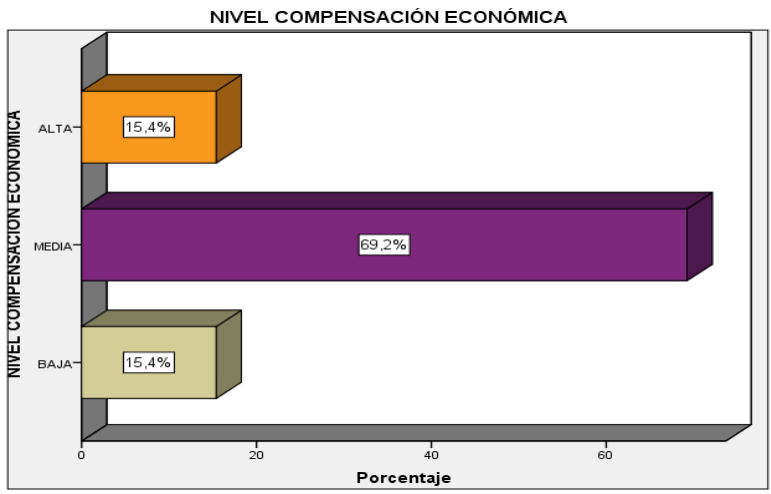

Fuente: Cuestionario satisfacción laboral docente de la FENUNCP 2016.

Los resultados que se observan en el grafico anterior muestran que el $69.2 \%$ de docentes refieren un grado de satisfacción media, considerando que el $100 \%$ de docentes encuestados son nombrados y existe puntualidad en el pago. Sin embargo, en relación a la satisfacción por la compensación económica la totalidad de ellos se sienten insatisfechos al no poder cubrir su presupuesto familiar.

\section{Dimensión: Condiciones de trabajo}

\section{Gráfico 8}

Condiciones de trabajo del personal docente de la Facultad de Enfermería de la UNCP 2016 NIVEL DE CONDICIONES DE TRABAJO

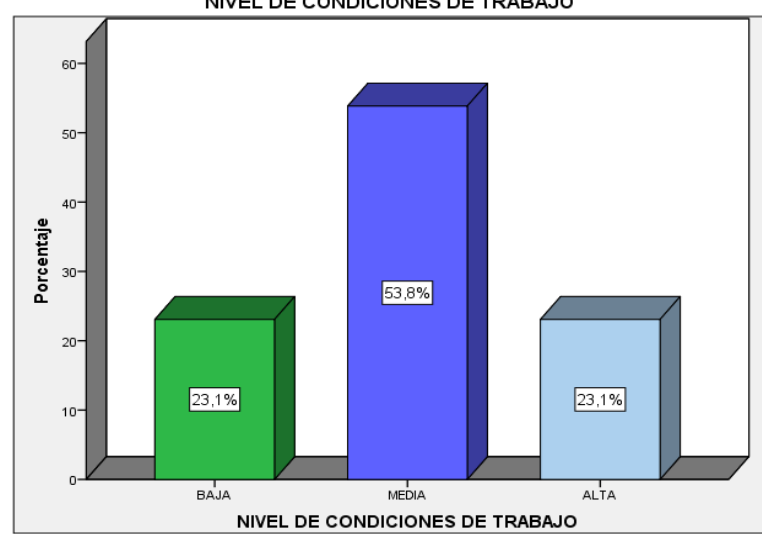

Fuente: Cuestionario satisfacción laboral docente de la FENUNCP 2016.

Para un adecuado proceso enseñanza - aprendizaje es necesario contar con espacios físicos e implementación adecuada, en este sentido las condiciones de trabajo juegan un papel importante, la satisfacción del docente en relación a las condiciones de trabajo es del $23.1 \%$ baja, $53.8 \%$ media y $23.1 \%$ alta, existiendo variedad de opiniones.

\section{Productividad del docente de la Facultad de Enfermería}

Gráfico 9

Productividad en investigación de los docentes de la Facultad de Enfermería de la UNCP 2016.

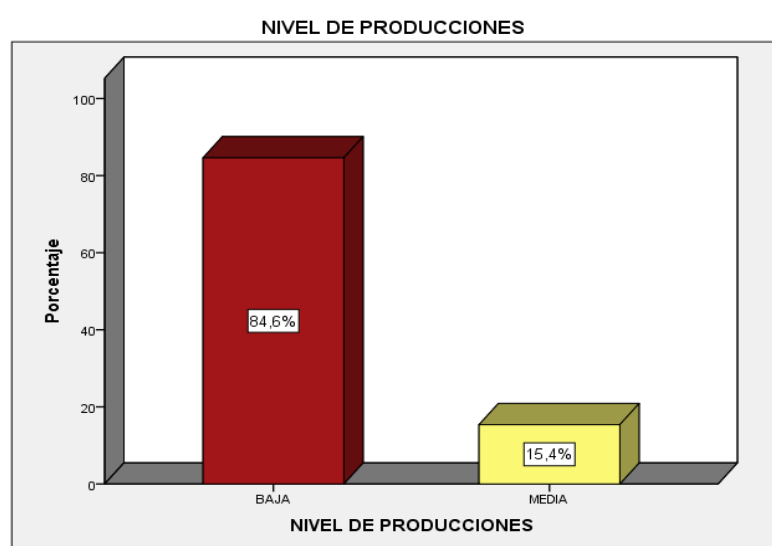

Fuente: Cuestionario satisfacción laboral docente de la FENUNCP 2016.

Existe baja productividad en el $84 \%$ de docentes. Está referida a la no publicación de los resultados, siendo que 
los docentes en los últimos cinco años si han producido investigaciones en promedio de uno por año, pero lo que no se hace probablemente por cultura de difusión es la no publicación de artículos en revistas locales o institucionales mucho menos en revistas indexadas o de gran prestigio.

\section{Gráfico 10}

Productividad y condiciones de trabajo de los docentes de la Facultad de Enfermería de la UNCP 2016.
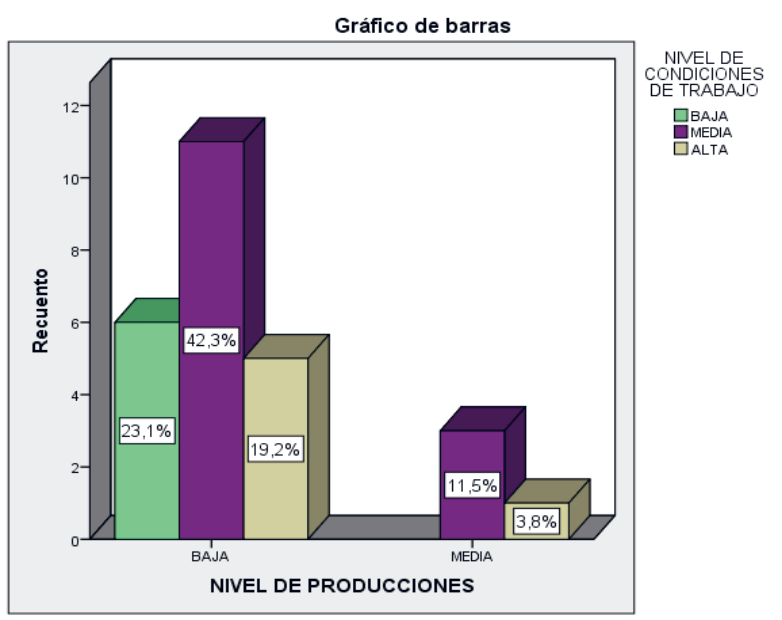

Fuente: Cuestionario satisfacción laboral docente de la FENUNCP 2016.

Al revisar los hallazgos de productividad y las condiciones de trabajo; se puede apreciar que la mayoría de docentes considera que existen bajas condiciones de trabajo; esto puede estar influenciando la baja productividad del docente.

\section{Gráfico 11}

Productividad y capacitación - perfeccionamiento de los docentes de la Facultad de Enfermería de la UNCP 2016.

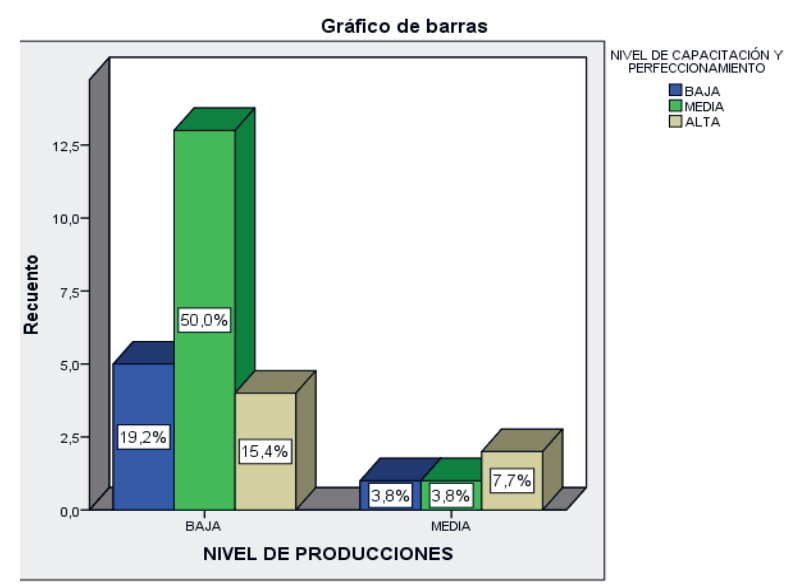

Fuente: Cuestionario satisfacción laboral docente de la FENUNCP 2016.

Referente a la capacitación y perfeccionamiento de los docentes, el gráfico muestra que no hay relación entre los que están capacitados y/o han realizado estudios de perfeccionamiento y la productividad.

\section{Factores que dificultan o facilitan la producti- vidad del docente}

\section{Gráfico 12}

Políticas de investigación de la universidad como factor que favorece/limita la productividad en investigación.

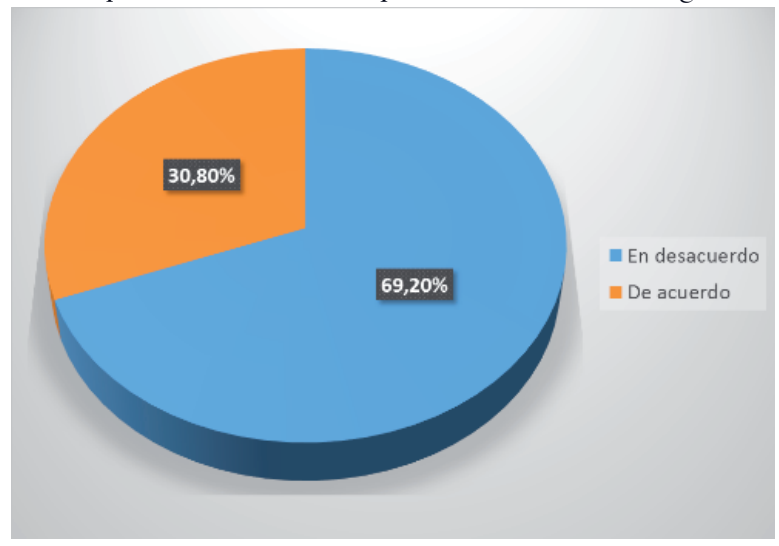

Fuente: Cuestionario productividad del docente de la FEN 2016.

El $69 \%$ de docentes considera que las políticas de investigación en la UNCP no están implementadas y que requieren establecerse políticas y lineamientos de investigación para revertir la baja productividad del docente.

\section{Gráfico 13}

recursos financieros que ofrece la universidad para favorecer la productividad en investigación.

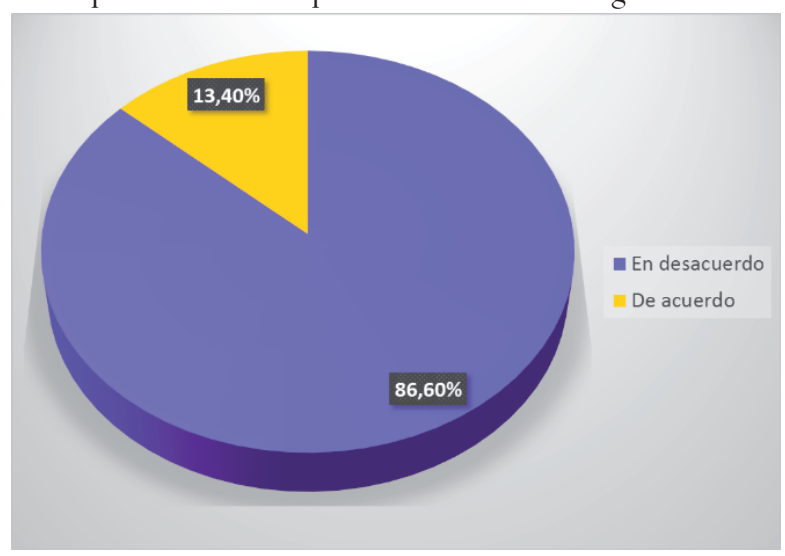

Fuente: Cuestionario productividad del docente de la FEN 2016.

Un factor de suma importancia es asignar los recursos financieros al desarrollo de las investigaciones; si bien, la UNCP asigna presupuesto en investigación, este parece no satisfacer a los docentes investigadores, toda vez que el $86 \%$ opina no estar de acuerdo con los recursos financieros que ofrece la universidad para este fin. Tomando en consideración la importancia que tiene movilizar recursos financieros externos e internos para asegurar el desarrollo de la investigación en la universidad, se debe reprogramar el presupuesto, ligado a las políticas y lineamientos en investigación debido a que las actividades de ciencia y tecnología revisten medular importancia para la investigación y desarrollo experimental. 


\section{Gráfico 14}

Oportunidades de capacitación en investigación que brinda la universidad a los docentes

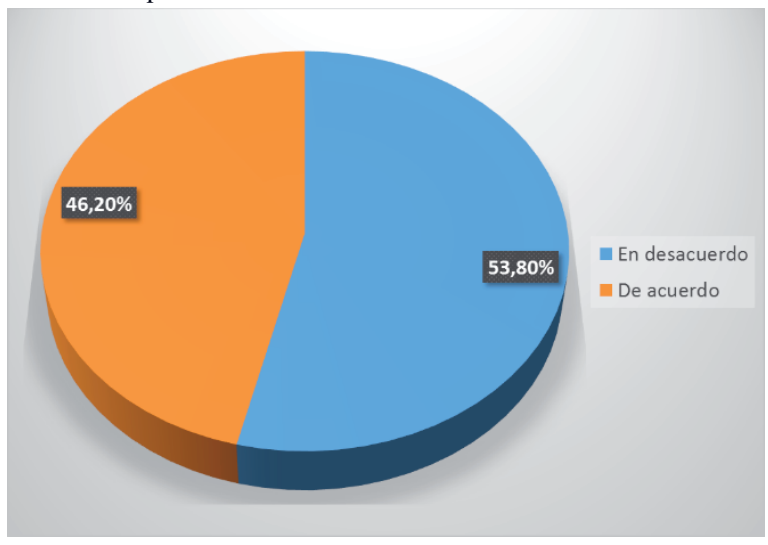

Fuente: Cuestionario productividad del docente de la FEN 2016.

Las universidades, como organización de educación, deben enfrentar la formación y capacitación de su personal como algo prioritario; el cual, además de los recursos materiales, financieros y/o administrativos, es el capital más dinámico que toda organización necesita para llevar a cabo sus procesos, como manifiesta Diez y Abreu (2009). Al respecto un 53 $\%$ de docentes expresan que la universidad no está brindando las oportunidades de capacitación en investigación.

Satisfacción laboral y productividad de los docentes de la Facultad de Enfermería de la UNCP

Tabla 1

Productividad y nivel de satisfacción de los docentes de la Facultad de Enfermería de la UNCP 2016.

\begin{tabular}{|c|c|c|c|c|}
\hline \multirow{2}{*}{$\begin{array}{c}\text { NIVEL DE } \\
\text { PRODUC- } \\
\text { CIONES }\end{array}$} & \multicolumn{3}{|c|}{ NIVEL DE SATISFACCIÓN } & \multirow{2}{*}{ Total } \\
\hline & BAJA & MEDIA & ALTA & \\
\hline \multirow[t]{2}{*}{ BAJA } & 7 & 8 & 7 & 22 \\
\hline & $87,5 \%$ & $80,0 \%$ & $87,5 \%$ & $84,6 \%$ \\
\hline \multirow{2}{*}{ MEDIA } & 1 & 2 & 1 & 4 \\
\hline & $12,5 \%$ & $20,0 \%$ & $12,5 \%$ & $15,4 \%$ \\
\hline \multirow{2}{*}{ Total } & 8 & 10 & 8 & 26 \\
\hline & $100,0 \%$ & $100,0 \%$ & $100,0 \%$ & $100,0 \%$ \\
\hline
\end{tabular}

Fuente: Cuestionario satisfacción laboral docente de la FENUNCP 2016.

\section{Gráfico 15}

Nivel de producciones y nivel de satisfacción del personal docente de la Facultad de Enfermería de la UNCP 2016.

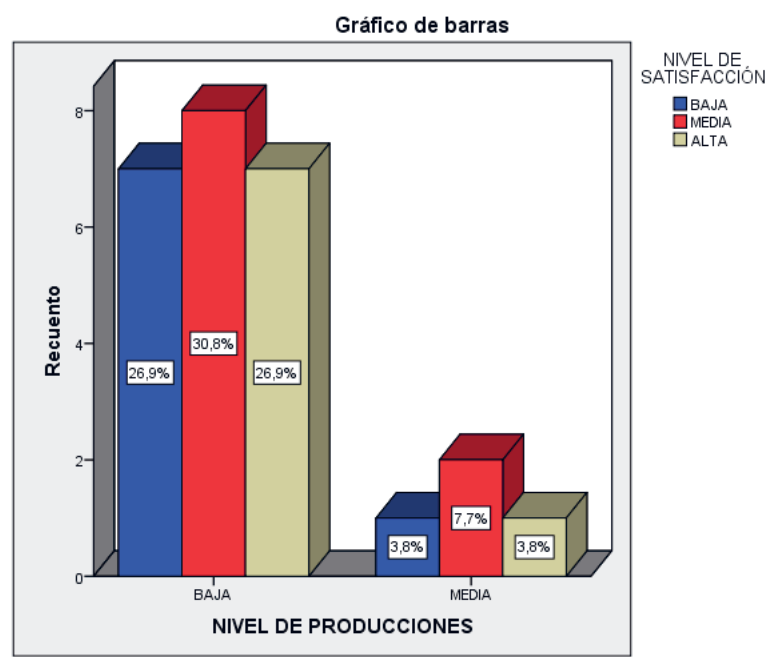

Fuente: Cuestionario satisfacción laboral y productividad del docente de la FEN 2016.

El resultado encontrado en la relación de Satisfacción laboral con la productividad del docente de la Facultad de Enfermería de la UNCP; se tiene, que del $100 \%$ de los docentes encuestados, el $38 \%$ tiene una satisfacción media, en lados opuestos están centrados el $30 \%$ de docentes que tienen una satisfacción baja y el $30 \%$ tiene una satisfacción alta. Por tanto, se observa que existe una productividad baja en los docentes independientemente de la satisfacción baja, media o alta, igual la mayoría tiene una productividad baja.

\section{Prueba de hipótesis}

La hipótesis general planteada fue:

$\mathbf{H}_{1}$ : Existe relación entre la satisfacción laboral y productividad en docentes de la Facultad de Enfermería de la UNCP.

Ho: No existe relación entre la satisfacción laboral y productividad en docentes de la Facultad de Enfermería de la UNCP.

\section{Discusión}

La satisfacción laboral definida como la actitud del trabajador, basado en las creencias y valores que el trabajador desarrolla en su propio trabajo, son determinadas conjuntamente por las características actuales del puesto como por las percepciones que tiene el trabajador de lo que "deberían ser”. Al respecto Gamero (2003)' refiere que: "La satisfacción o insatisfacción laboral están relacionados con muchos comportamientos y resultados que son de gran trascendencia para los propios individuos, para la organización o para la sociedad"

El éxito de una organización en este caso la universidad está estrechamente relacionado con el grado de conformidad de los que trabajan en ella. Los resultados obtenidos muestran en general que la satisfacción laboral en 
los profesionales docentes en un $38.5 \%$ de la Facultad de Enfermería de la UNCP es de nivel media, existiendo polaridad en los demás docentes siendo que el $30 \%$ tiene insatisfacción o satisfacción baja y el $30 \%$ satisfacción alta. Consideramos que las autoridades deberían conocer, la importancia que tienen los recursos humanos para el éxito de la institución. Los docentes en este caso son el recurso más preciado para generar ciencia y tecnología, al depender de ellos, en un alto porcentaje, el buen funcionamiento y desarrollo de la organización.

Para la satisfacción laboral, es importante tener en cuenta los factores intrínsecos que comprenden el nivel de independencia que tiene un docente, la libertad de catedra, las distinciones y reconocimientos, la seguridad del puesto, el estatus que tienen y la calidad de relaciones interpersonales que tienen los docentes con sus autoridades, En este estudio encontramos, que la mayoría de ellos tienen un nivel medio de satisfacción, al igual que los factores extrínsecos como las condiciones de trabajo, la capacitación y perfeccionamiento; también existe un nivel medio de satisfacción de los docentes con los factores intrínsecos. En relación a la remuneración económica hay un descontento general y bajo nivel de satisfacción.

Otra de las variables estudiadas fue la productividad que tienen los docentes; la motivación juega aquí un papel importante y está referido al desempeño realizado por las personas en su puesto de trabajo, es fundamental para lograr día a día las metas de la organización, que el capital humano sea considerado como el principal elemento de la institución. Lo deseable es que todos los docentes sean realmente productivos, que se comprometan e impliquen al máximo y sean eficientes en su trabajo. Sin embargo, esto no se observa en los docentes al presentar un nivel bajo de productividad, referido a la publicación de artículos, esto depende de muchos factores, como su formación, pero también, y en especial, de factores de tipo motivacional.

Si se parte del hecho que, el buen funcionamiento de la universidad depende en un gran porcentaje del ánimo o el empeño de los docentes; la influencia de los directivos sobre los trabajadores tiene como objetivo esencial hacer cumplir la misión y los objetivos organizacionales emanados de la UNCP y esto, desde luego, lleva a concentrar cada vez más los esfuerzos en la atención al hombre, de manera que se puede alcanzar su satisfacción; entendida ésta como la sensación que el docente siente al lograr el equilibrio entre sus necesidades o grupo de necesidades y el objeto o los fines de la universidad. Claro está, que algunas veces se pueden encontrar docentes que se dedican única y exclusivamente a terminar sus tareas, sin preocuparse de la calidad de las mismas, afectando de grave manera al desarrollo de la universidad.

A veces resulta difícil distinguir entre la motivación y la satisfacción laboral, debido a su estrecha relación. De ahí, que la satisfacción o insatisfacción con el trabajo dependa de numerosos factores, como el ambiente físico donde trabaja, el hecho de que el jefe lo llame por su nombre y lo trate bien, el sentido de logro o realización que le procura el trabajo, la posibilidad de aplicar sus conocimientos, que la docencia le permita desarrollar nuevos conocimientos y asumir retos. Concordamos con Monteza (2012), cuando, después de su estudio, afirma que el clima laboral y la satisfacción laboral son pilares fundamentales en las organizaciones competitivas. Cualquier variación en éstos, afecta el ambiente de trabajo y forzosamente a los que lo integran.

En relación a la baja productividad presentada por los docentes, como resultado de la investigación, se puede citar diferentes factores que probablemente sean visibles para este resultado, cabe también señalar algunos obstáculos que limitan la producción de conocimientos en las universidades y otros aspectos que limitan la acción investigadora de las universidades y, sus efectos en el quehacer investigativo de los docentes. Autores como Padrón (1999a), Aponte (2001) y Brunner (2000), coinciden en señalar entre otros aspectos: la escasez de financiamiento, desconexión de la problemática social, así como de otras áreas o estructuras de procesos, la vinculación de las investigaciones a intereses personales y modas paradigmáticas, falta de apoyo institucional, incipiente divulgación e intercambios de productos investigativos, poca atención a la concepción de productividad de los procesos de investigación y escasa participación del sector productivo en el desarrollo de investigaciones. Cabe considerar que la escasa formación en investigación, según Domínguez (2004), es otro aspecto que influye negativamente en la producción de conocimientos. Debe señalarse que la formación es el instrumento clave que posibilita el procesamiento de la información con el propósito de generar conocimiento e innovación dentro de la organización, a fin de responder a sus necesidades de desarrollo continuo, cambio o mejora. Mogollón (2007), indica que la actividad investigativa tiene como finalidad desarrollar la formación operativa y permanente del investigador de este siglo, sustentada en la producción de conocimientos dirigidos al desarrollo de potencialidades intelectuales y académicas de alto nivel mediante el uso de principios que se complementan, denominados: universalidad, democracia, innovación y pertinencia social, equidad y calidad (Narváez y Burgos, 2011).

A estos principios, deben responder el compromiso que tienen los docentes investigadores frente a la sociedad; encargados de fortalecer, mejorar o proponer alternativas de solución frente a los principales problemas de salud de la región y el país. 


\section{Conclusiones}

- Los docentes de la Facultad de Enfermería en su mayoría $(38 \%$ ) tienen un nivel medio de satisfacción laboral, y el $30 \%$ tienen un nivel bajo de satisfacción frente al $30 \%$ que tiene un nivel alto de satisfacción laboral.

- En relación a los factores extrínsecos se tiene que la mayoría de docentes (38\%) tienen un nivel medio de satisfacción, en las dimensiones: condiciones de trabajo, capacitación y perfeccionamiento y reconocimiento y distinciones tienen un nivel medio de satisfacción.

- Referente a los factores intrínsecos se tiene que la mayoría (38\%) tiene un nivel medio de satisfacción, en las dimensiones como independencia, libertad de cátedra, relaciones interpersonales la mayoría de docentes tienen un nivel medio de satisfacción.

- La edad y el tiempo de servicio, la condición laboral; así como, la dedicación y los grados obtenidos no fueron relevantes para la satisfacción laboral en este estudio, indistintamente en cada grupo etáreo y dependiendo de su tiempo de servicio existe un nivel medio de satisfacción laboral.

- La productividad en investigación de los docentes de la Facultad de Enfermería es bajo, si bien se realiza investigaciones anualmente la debilidad está en la publicación de los mismos en revistas indexadas o de prestigio, siendo escasa o nula la publicación en muchos casos.

- Se ha comprobado que no existe relación entre la satisfacción laboral de los docentes y la baja productividad que tienen, independientemente del nivel de satisfacción la mayoría tienen baja productividad.

\section{Referencias bibliográficas}

Belkis Jiménez de Vargas Bravo, M. (2001). Productividad en investigación del docente universitario. Disponible en: http://www.revistaespacios.com/ a93v14n03/40931403.html\#*

Álvarez, R. (1984). Universidad: Investigación y productividad. Ediciones Paral. Caracas, 226 p.

Arnao de U., D. (1984). Hacia una forma distinta de valorar las actividades de investigación en Venezuela. CONICIT. O.E.A. CENDES. Caracas, 72 p.

Ávila, J. (1982). La productividad de nuestra investigación biomédica. En: los objetivos y la productividad de nuestra investigación biomédica. Publicaciones de la Facultad de Medicina, U.C.V. NEIR Contreras, pp. 73-76.

Bravo C., \& Jiménez-Figueroa A. (2011). Bienestar psicoló- gico, apoyo organizacional percibido y satisfacción laboral en funcionarios penitenciarios de Chile. Revista Esp. Sanid Penit, Universidad de Talca, Chile.

Bain, R. (2003), La productividad. 2a . Edición. Editorial McGraw Hill. Colombia

Galaz J. (2002). La satisfacción en el trabajo de académicos en una universidad pública estatal. Revista Perfiles Educativos, México. Instituto de Investigaciones sobre la Universidad y la Educación. México.

Gaither, N. y Frazier, G. (2000), Administración de producción y operaciones. México International Thomson Editores.

Hellriegel D. \& Slocum Jr. J. (2004). Comportamiento organizacional. Los individuos en las organizaciones, México, Ed. Thomson

Jiménez DE V., B. (1993). Evaluación de la productividad en investigación del docente universitario. Universidad "Simón Bolívar". Camurí Grande. Mimeografiado. $54 \mathrm{p}$.

Keith D., J. (2002). Comportamiento humano en el trabajo. Actitudes de los empleados y sus efectos. México: MCGraw-Hill.

Lindarte, E. (1978). Determinantes del desempeño entre investigadores de la región centro occidental. CONICIT FUDECO. Barquisimeto, 160 p.

López M. (2009). Consecuencias sicosociales en del trabajo en personal de enfermería como indicadores subjetivos de rendimiento desde el enfoque de la gestión de recursos humanos. Tesis Doctoral. Universidad de Murcia, España.

Márquez M. (2002). Satisfacción laboral. Boletín electrónico AEG, publicación abril. Asociación de Egresados y Graduados de la Pontificia Universidad Católica del Perú.

Núñez B., Miguel (2007). Material de apoyo del seminario Gestión de la Productividad. Doctorado en Ciencias de la Ingeniería, mención Productividad. Universidad Nacional Experimental Politécnica "Antonio José de Sucre”. Barquisimeto, Venezuela.

Peña y Lillo. A. DE S/F. Factores que afectan la productividad científica de las unidades de investigación. CONICET. Buenos Aires. 20 p.

Rivas E., \& López F. (2011). Psicología social de las organizaciones. Centro de Documentación de Estudios y Oposiciones, CeDe. 130. Recuperado de: http://www. 
pir.es/temas_muestra_2011/11.SOCIAL.pdf,

Robbins S. (2004). Comportamiento organizacional.Valores, actitudes y satisfacción con el puesto. México: Pearson Education.

Robbins, S. y Coulter, M. (2005), Administración. México. Prentice-Hall Hispanoamericana, S.A.

Roche, M. (1981). La Productividad de nuestra investigación biomédica. Publicaciones de la Facultad de Medicina. U.C.V. NEIR Contreras, Caracas, pp. 70-72.

Sánchez S., Artacho. C., Fuentes F., \& López-Guzmán, T. (2007). Análisis de los determinantes estructurales de la satisfacción laboral. Aplicación en el sector educativo. Estudios de economía aplicada, Área de Organización de Empresas Universidad de Córdoba.

Solanes A., \& Zaragoza B. (2010). Trabajo de preferencia o contrato indefinido? Un análisis de su impacto sobre el estado del contrato psicológico. Revista Acciones e Investigaciones Sociales.

Salomón Y, Benítez L, Saladrigas H, Martínez A. Análisis métrico de la producción científica en comunicación social en Cuba. Rev. Acimed. 2006; Vol.14 Nº4. [En línea]. URL disponible: http://dialnet.unirioja.es/servlet/ tesis?codigo $=18928$.

Jiménez B. Productividad en investigación del docente universitario. Rev. Espacios. 1993; Vol. 14 N³. [En línea]. URL disponible: file: / / C: /Documents $\% 20$ and\%20Settings / Propietario/Escritorio/tesis / tesis\%20biblio/productividad\%20del\%20docente\%20universitario.htm.

Favero R. Evaluación de publicaciones periódicas científicas y técnicas brasileñas. Rev. Acimed 1998; Vol 9 N²4. [En línea]. URL disponible:www.bvs.sld.cu/revistas/ aci/vol9_s_01/sci10100.htm.

Luiz A. Análisis de la producción científica en biblioteconomía y documentación en el Período1995/2004: estudio en los principales programas españoles y brasileños. Tesis doctoral, Getafe (Madrid), 2007. [En línea]. URL disponible: http://earchivo.uc3m.es/bitstream/10016/2536/1/Tesis_Adilson.pdf.

El Plan Operativo Institucional de la UNMSM para el año 2010. [En línea]. URL disponible: http://www. unmsm.edu.pe/transparencia/archivos/plan_ operativo_institucional_2010_POI.Pdf

Pasache Herrera Evelyn Roxana y Torres Vásquez Lissette Estefany (2011). La producción científica de los docentes obstetras de la E.A.P. de Obstetricia - UNMSM, 1999-2010.

Escobar-Pérez Bernabé, García-Meca Emma, Larrán-Jorge Manuel (2013). Factores que influyen sobre la producción científica en Contabilidad en España: la opinión de los profesores universitarios de Contabilidad (II parte). Revista Española de Documentación Científica 37(2), abril-junio 2014, e047. ISSN-L: 0210-0614. doi: http://dx.doi.org/10.3989/ redc. 2014.2.1087

Albaro Chabés y Suárez y col. Diagnóstico de la investigación biomédica en el Perú

Comunicación Preliminar. Anales de la Facultad de Medicina. Vol. 58, Nº3 - 1997 [Enlínea]. URL disponible: <http://sisbib.unmsm.edu.pe/BvRevistas/ anales/v58_n3/diagn\%C3\%B3stico_inves.htm>. ISSN $1025-5583$.

Narváez Serra, Jellicy; Burgos Tovar, José. La productividad investigativa del docente universitario Orbis. Revista Científica Ciencias Humanas, vol. 6, núm. 18, enero-abril, 2011, pp. 116-140 Fundación Miguel Unamuno y Jugo. Maracaibo, Venezuela

Cáceres Castellanos, Gustavo. La importancia de publicar los resultados de Investigación. Fac. Ing. [online]. 2014, vol.23, n.37 [citad 2016-1210], pp.7-8. Available from: <http://www. scielo.org.co/scielo.php?script $=$ sci_arttext\&pid=S0121-11292014000200001\&lng=en\&n$\mathrm{rm}=\mathrm{iso}>$. ISSN 0121-1129.

Diez, Jennifer y Abreu José Luis. Impacto de la capacitación interna en la productividad y estandarización de procesos productivos: un estudio de caso. Daena: International Journal of Good Conscience. 4(2): 97-144. Septiembre 2009. ISSN 1870-557X.Disponible en: http: / / www.spentamexico.org/v4-n2/4(2)\%20 97-144.pdf

Ascencio Jordán Erika del Pilar, Navarro Espinosa Johanna Andrea (2015). Importancia de la capacitación y el desarrollo del talento humano en el ecuador. Universidad Tecnológica Ecotec. Observatorio Economía Latinoamericana. ISSN: 1696-8352. Disponible en: http://www.eumed.net/cursecon/ecolat/ ec/2015/capacitacion.html

Carvajal, Lizardo (2013). Los recursos en la investigación científica y sus clases. Disponible en: http://www. lizardo-carvajal.com/los-recursos-en-la-investigacion-cientifica-y-sus-clases / 
Pérez, J., Méndez, S., Jaca, M. (2010). Motivación de los empleados:Teoría de Herzberg (en español). Sevilla,

Brunner, J. (2000). Evaluación y financiamiento de la educación Superior en América Latina. En CINDA: Acreditación Universitaria para América Latina. CRESALC/ UNESCO.

Aponte, C. (2001). Tendencias de las investigaciones en el colegio universitario Francisco de Miranda. Caracas. Producciones -en Línea Disponible: http://lineai.entretemas.com/ ArticulosAnteriores/index.htm

Padrón, J. (1999:a). El problema de organizar la investigación. Línea de investigación y enseñanza de aprendizaje de la investigación. Línea-I-Caracas. [En línea], Disponible: http://www.lineai.org

Domínguez, G. (2004). La Generación de conocimiento en la organizaciones educativas mucho más que una demanda social: El gran reto y aportación de la pedagogía y de la formación al campo de la organización. En Actas del $8^{\circ}$ Congreso Interuniversitario de Organización de Instituciones Educativas. Sevilla, noviembre.

Mogollón de G., A. (2007). Formación del investigador universitario. Revista Ciencias De La Educación. Año 61 Vol. 11 No 29 l Valencia, enero-junio. PP. 217-230.

Monteza Chanduvi, Nancy Elizabeth (2012). Influencia del clima laboral en la satisfacción de las enfermeras del centro quirúrgico Hospital Essalud. Tesis Pos grado. Perú: Chiclayo.

Gamero Burón, Carlos (2003). Satisfacción laboral de los asalariados en España. Especial referencia a las diferencias por género. Universidad de Málaga Facultad de Ciencias Económicas y Empresariales Dpto. de Estadística y Econometría Campus Universitario El Ejido, s/n 29013 Málaga e-mail: gamero@uma.es 